\title{
Genetic algorithm for vertical handover (GAfVH) in a heterogeneous networks
}

\author{
Iman Zubeiri, Yasmina El Morabit, Fatiha Mrabti \\ Signals, Systems and Components laboratory, Faculty of Science and Technologies, \\ University Sidi Mohamed Ben Abdellah Fez, Morocco
}

\begin{abstract}
Article Info
Article history:

Received Jan 19, 2018

Revised Jul 22, 2018

Accepted Mar 4, 2019

Keywords:

$5 \mathrm{G}$

Genetic algoritm

Radio access techonologies

Vertical handover

ABSTRACT

The fifth generation $(5 \mathrm{G})$ wireless system will deal with the growing demand of new multimedia and broadband application. The $5 \mathrm{G}$ network architecture is based on heterogeneous Radio Access Technologies (RATs). In such implementation the Vertical handover is a key issue. Up till now, systems are using simple mechanisms to make handover decision, based on the evaluation of the Received Signal Strength (RSS). In some cases these mechanisms are not efficient. This paper presents a new vertical handover algorithm based on Genetic Algorithm (GAfVH). It aims to reduce the number of unnecessary handovers, and optimizes the system performance. We compare our simulation results to the Received Signal Strength (RSS) based method. The results show that the number of handovers decreases. Moreover, we demonstrate that the network selection result can differ from an application to another.
\end{abstract}

Copyright (c) 2019 Institute of Advanced Engineering and Science. All rights reserved.

\section{Corresponding Author:}

Iman Zubeiri,

Signals, Systems and Components laboratory,

Faculty of Science and Technologies,

University Sidi Mohamed Ben Abdellah Fez, Morocco.

Email: iman.zubeiri@usmba.ac.ma

\section{INTRODUCTION}

The fifth generation $(5 \mathrm{G})$ network is the next telecommunication standard beyond $4 \mathrm{G}$ and IMT advanced. It aims to achieve a higher capacity, a peak data rate over $10 \mathrm{Gbps}$ and a low latency $<1 \mathrm{~ms}$. The $5 \mathrm{G}$ includes some new research fields like Millimeter-Wave, Massive MIMO and Dense Network. It offers some new use case such as Vehicle to Everything (V2X), Healthcare application, and 3D Television (3DTV).

The 5G network architecture is based on heterogeneous Radio Access Technologies (RATs). In a heterogeneous environment, Vertical Handoff $(\mathrm{VH})$ is an essential issue. It means handover among different standards. Up till now, systems are still using simple mechanisms to make handover decision, based on the evaluation of the Received Signal Strength (RSS). In some cases these mechanisms are not efficient, for example we can find a system with a good RSS and a worse data rate.

In these cases, unnecessary handovers may occur and cause high signaling overhead and system performance degradation. Therefore, efficient Radio Access Technologies (RAT) selection algorithms have to be investigated. This paper presents a new vertical handover algorithm based on Genetic Algorithm. Our scheme is a Genetic Algorithm for Vertical Handover (GAfVH), It makes a handover decision based on the genetic algorithm selection results. It aims to reduce the number of unnecessary handovers, and optimizes the system performance. We define four important network selection features for our GA: the Received Signal Strength (RSS), the Data-Rate, the Bit-Error-Rate (BER), and the Delay. The GA selects a chromosome, which is composed from the best genes, DR, RSS, BER, Delay. These chromosomes values are compared with the measured values obtained from the network. If the measured value is less significant than the best value, the algorithm decides to handoff from the current network. Using simple VH mechanisms 
such as the RSS based method presented many disadvantages. The first one is making a VH decision based on the comparison of the RSS measurements. What drives the UE to make a lot Handovers, each time when the RSS of a network, becomes superior to another. What about, if this RSS value is not too bad and still effective?

Besides, the second disadvantage, is taking into account only the RSS parameter, it's may not be efficient to perform a VH. Since a good signal quality can be reached near to an Access Point (AP) and the Wireless interface is activated the UE is automatically connected to this wireless network. But what if this network is not working?

Our solution, GAfVH, uses the best values of the RSS, obtained from the GA as a threshold and compare it with the measured value. Even if the RSS of a network becomes better than another, if it is not superior to the threshold value there is no need to make a handover decision. Noting that, the GafVH algorism is based on multiples criteria, the handover decision will be based on multiple parameters.

This paper is organized as follow; section two presents related works, section three presents the Genetic Algorithm and the proposed vertical handoff algorithm (GAfVH), section four presents the simulation results. Finally we conclude in section five

\section{RELATED WORKS}

Various methods and approaches of network selection have been discussed in the literature. A network selection scheme based on weight estimation of QoS parameters in Heterogeneous wireless multimedia network is proposed in [1]. This scheme estimated the weights of QoS parameters such as bandwidth, delay, packets loss and data transfer cost, by using similarity to ideal solution. The results showed that the proposed algorithm can select the best network in a Heterogeneous environment. Authors in [2] proposed an analytical model to capture the preferences of end-users, using this model they designed an Automatic Network Selection (ANS) mechanism that took into account all aspect of the trade-off, between the quality of the connection, the preferences of the users, and the cost. This model was implemented and tested in a multi technologies simulator. Results showed that the proposed solution can be beneficial to both users and operators. A new intelligent vertical handoff decision algorithm using Fuzzy Logic and Generic algorithm was proposed in [3]. It estimates the handoff requirement to select the optimal network and makes a handoff decision.

In [4], a multi-criteria access network selection algorithm was proposed in WIMAX environment, in order to provide high quality of services and satisfy different types of users. This algorithm is based on a combination of Analytical Hierarchy Process [5], and Grey Relational Analysis [6]. The AHP method decided on the relative weights of the criteria set, according to networks performances. The GAR ranked the network alternatives. A novel approach for network selection was proposed in [7], it is based on AHP method and Bankruptcy Game. In this approach the AHP method evaluated the weights of multiple decision criteria, than the Bankruptcy Game is used to assess the potentials of available candidate networks. Another network selection mechanism as presented in [8], was based on two decision making methods, the Multi-AHP and GRA. Multi-AHP is used to weigh criterions while GRA ranked the alternatives. In [9] the authors proposed a new ranking alghorithm which combine Multi-Attributes Decision Making [10] and Mahalanobis distance. Firt they applaied a classification method to builde classes which have homogenous criteriat. Then the Fuzzy AHP and MADM methods are applied to determine both the weigths of interclasses and intra-classes, finally Mahalanobis distance is used to rank the alternatives. In [11] a brand new approach for network selection was proposed. It used MADM as a ranking algorithm followed by Entropy to initialized weight.

\section{THE AGFVH ALGORITHM}

\subsection{Genetic Algorithm}

Genetic algorithm (GA) is a metaheuristic inspired by the mechanism of natural selection. It used to generate high quality solutions in order to optimize a search problem. The main advantage of the GA is its fast convergence. It can converge quickly on a problem's specific solution [12]. Another advantage is that the GA does not provide a single solution but a list of optimum solutions. The GA chromosome is represented by a simple data structure vector. This structure has different data types which define their genes. In the case of RAT selection the chromosome can have many parameters as genes, we chose the most important of them which are the DR, RSS, BER Delay.

The Genetic Algorithm can be implemented using the following steps:

1. Initialization: we randomly generated an initial population of ' $n$ ' chromosomes.

2. Fitness measures: we evaluate the fitness of the initial population's chromosomes. 
3. Construction of a new population: using the following steps we reproduce the new generation.

a. Selection: the selection process is based on the level of the chromosomes fitness.

b. Crossover: The objective of the crossover is to make new individuals for the incoming generation with a probability of crossover.

c. Mutation: The new created individual will be mutated at a definite point.

4. Stopping Criteria: the process is repeated until a desired optimum solution is reached.

\subsection{Implementation of genetic algorithm in network selection}

3.2.1. Constructing the chromosome structure

We consider four attributes: The Data-Rate (DR), the Received Signal Strength (RSS), Bit Error Rate (BER), and Delay, as shown in the Table 1.

Table 1. The chromosome structure

\begin{tabular}{llll}
\hline Gene & Coded bits & Range & Level \\
\hline Data rate & 7Bit & $0 \mathrm{Mbps} \sim 100 \mathrm{Mbps}$ & 00 \\
RSS & 6Bit & $-100 \mathrm{dbm} \sim-50 \mathrm{dbm}$ & 50 \\
Bit error rate & $3 \mathrm{Bit}$ & $10 \sim 10$ & 8 \\
Delay & 2Bit & $10 \mathrm{~ms} \sim 100 \mathrm{~ms}$ & 4 \\
\hline
\end{tabular}

Our chromosome structure consists of four genes, with different size. The DR gene contains 100 values from $0 \mathrm{Mbps}$ to $100 \mathrm{Mbps}$, which means 100 decimal values it can be coded on 7 bits. The RSS gene contains 50 decimal values, it's coded on 6 bits. The BER gene has 8 decimal values it can be coded on 4 bits, and the Delay gene has only 4 values it's coded on 2 bits as shown in Table 1.

\subsubsection{Initialization and fitness measure}

We randomly generated an initial population of ' $n$ ' chromosomes. Each chromosome is a combination of the four genes and it's coded on 19bits. We have to compute now the Fitness measure for each gene, followed by the fitness of the chromosome We have to compute now the Fitness measure for each gene, followed by the fitness of the chromosome. The fitness measure of the gene (i) is given by:

$$
f i\left\{\begin{array}{cl}
\frac{w_{i}\left|g_{i}-g_{i}^{d}\right|}{g_{i}^{d}}, & \left|g_{i}-g_{i}^{d}\right|<g_{i}^{d} \\
w_{i}, & \text { Otherwise }
\end{array}\right.
$$

We set the parameters g1, g2, g3 and g4 corresponding respectively to the DR, the RSS, the BER, and the Delay. $g_{i}^{d}$ is the required QoS parameter, while $w_{i}$ represents the weight parameter. The fitness value of the chromosome is obtained by:

$$
F=\sum_{i=1}^{4} f_{i}
$$

Constructing the new generation using genetics steps.

We use the Roulette Wheel [13] selection method, for each chromosome, we calculate probability of it's selection $\mathrm{p}_{\mathrm{i}}$, this probability is given by:

$$
p_{i}=\frac{f_{i}}{\sum_{i=1}^{4} f i}
$$

\subsubsection{Crossover and mutation}

The aim of crossover process is to exchange characteristics of any two chromosomes with each other to form two new chromosomes.There are several crossover techniques, in this paper we applied the 2-point crossover process as shown in Figure 1. The Mutation technique is applied on the child genes, by altering a binary bit of 0 to 1 or vice versa. 


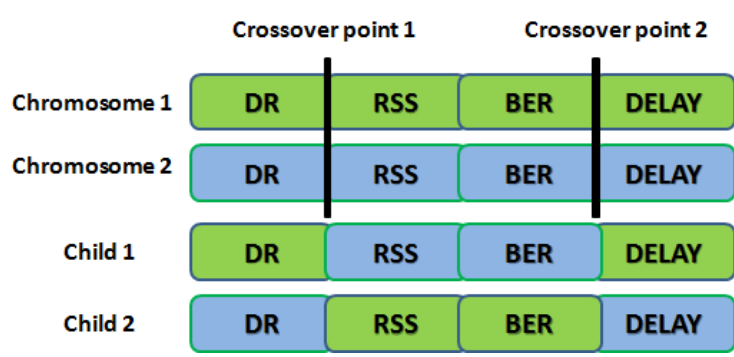

Figure 1. The crossover 2-point process

\subsubsection{The stopping criteria}

The optimum stopping criteria is when a best solution is reached, in our case the stopping criteria is when we obtain the best chromosome.

\subsection{The proposed vertical handover algorithm}

The proposed vertical handover algorithm is based on Genetic Algorithm as shown in Figure 2. The GA selects the best chromosome, which is composed from the best genes (DR,RSS, BER, Delay), this chromosome values are compared with the currents networks values which are the current (DR, RSS, BER and Delay), for each network. If the calculated value is less significant than the best value, the algorithm automatically decides to handoff from the current network, if not the handover does not occur. With the RSS based method the UE makes a Handover, each time the RSS of a network is better than another, even when this RSS value is not worst. The GAfVH uses the best values of the RSS, obtained from the GA as a threshold and compare it with the measured value. Even if the RSS of a network becomes better than another, if it's not superior to the threshold value there is no need to make a handover decision.

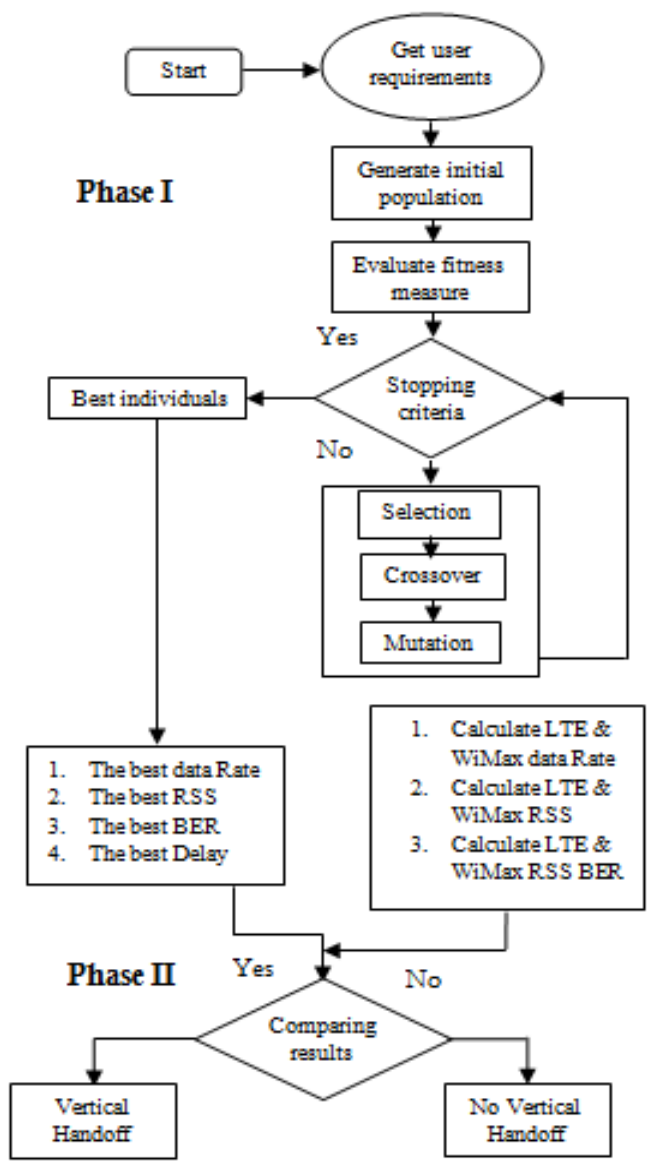

Figure 2. Graphic illustration of the GAfVH 


\section{SIMULATION}

In this section we are going to evaluate and establish a series of tests in order to assess the accuracy and the performance level of our proposed algorithm. The scenario consists of a heterogeneous network as shown in Figure 3, which integrate several networks, in our simulation we utilizes LTE and WiMax networks. For both LTE and WiMax networks we utilize OFDM modulation, for the LTE network the bandwidth is of 20Mhz.For the WiMax network the bandwidth is of $25 \mathrm{MHz}$ as shown in Table 2 . The eNodeBs and APs are deployed separately, and users moves in the network with different speeds that varies from $3 \mathrm{Km} / \mathrm{h}$ to $100 \mathrm{Km} / \mathrm{h}$. The GAfVH is implemented on the LTE eNodeB. It gets the measurements from the Heterogeneous environment and run the algorithm to decide when to handoff. The used Key Performance Indicator is: The occurred number of handovers.

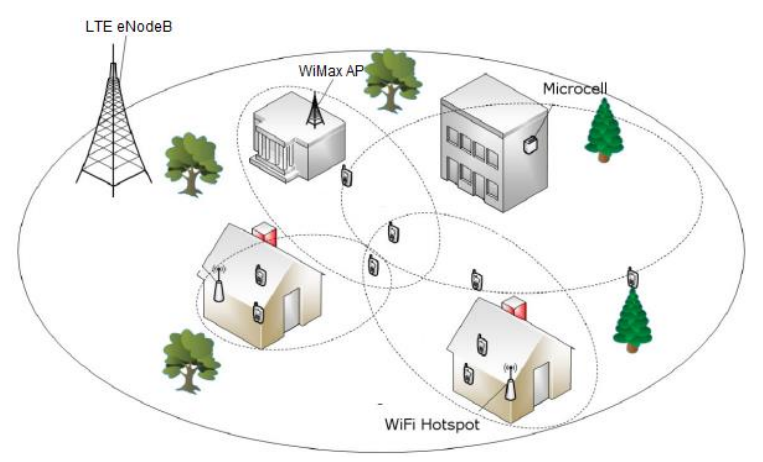

Figure 3. 5G heterogeneous network scenario

Table 2. The heterogeneous network parameters

\begin{tabular}{ccccc} 
Network & Modulation & Frequency & Number of MS & MS speed \\
\hline LTE & OFDM & $20 \mathrm{MHz}$ & $10 \sim 20 \sim 30$ & $10 \mathrm{~km} / \mathrm{h} \sim 50 \mathrm{~km} / \mathrm{h}$ \\
Wimax & OFDM & $25 \mathrm{MHz}$ & $10 \sim 20 \sim 30$ & $10 \mathrm{~km} / \mathrm{h} \sim 50 \mathrm{~km} / \mathrm{h}$ \\
\hline
\end{tabular}

In a first step we simulate a vertical handover with the RSS based method. This approach computes the RSS of the WiMax Access Point and the RSS of LTE eNodeBs, as shown in the Figure 4. Finally it compares the obtained values.

When the RSS of WiMax is better than the RSS of LTE, the UE switches to WiMax network. Then when the RSS of the LTE network becomes better the UE switch to it. The UE is connected to the LTE eNodeB. In Figure 4, at the times $t=40 \mathrm{~s}$, the RSS of WiMax becomes superior to the RSS of LTE, what drives the UE to switches to the WiMax network. Then in t=50s, the RSS of the WiMax network becomes lower than the LTE RSS which drives the UE to switch to the LTE network. In $t=60$ s the RSS of WiMax becomes superior, the UE has to makes a third handover to the WiMax Network. Another Handover at $\mathrm{t}=70 \mathrm{~s}$ when the RSS in LTE becomes superior and so the UE has to make a fourth handover to the WiMax network. With the RSS based method the UE makes a Handover, each time the RSS of a network is better than another, even when this RSS value is not worst. The GAfVH algorithm computes a best value of RSS relying on the genetic algorithm. This value is considered as a threshold. Then it calculates the RSS at both WiMax and LTE networks, these values are compared with the threshold. First the UE is connected to the LTE eNodeB, in Figure 5, when the RSS of the WiMax system becomes better than the calculated threshold the UE moves to the WiMax system. Second, when the RSS of the WiMax system becomes worst than the threshold the UE moves the LTE system. The GAfVH algorithm provides considerable gain, it reduces the number of unnecessary handovers and optimizes the system performance.

By using a VH algorithm based on RSS measurements, if we are near to an AP, for example, the UE is automatically connected to this network if the Wireless interface is activated, even when this network has no internet connection. In this case using only the RSS parameter to make a handover decision is not efficient. Noting that, the GAfVH algorism is based on multiples criteria which are mentioned previously, the handover decision will be based on multiple parameters. First we are going to evaluate a VH based on the DR parameter. We calculate the throughput of both the LTE and the WiMax networks, in a case of a low number of user per cell the throughput of the WiMax network is better, so the UE is automatically connected to the WiMax network as shown in Figure 6 If the number of the connected users increases in the WiMax system 
the UE switches to the LTE network as shown in Figure 7. In a third step, the simulations are based on the BER calculations. The UE is connected to the WiMax Network, when the BER of the LTE network becomes better than the best BER, the UE handoff from the WiMax network and connects to the LTE network as shown in Figure 8.

In Figure 9 we illustrate the performance of the RSS based method along with the GAfVH algorithm, in term of the occurred handover number. The GAfVH algorithm tends to minimize the handover number. However our algorithm makes two handovers instead of four. The GAfVH algorithm tends to minimize the handover number. However our algorithm makes two handovers instead of four. Moreover, the network selection process can differ from an application to another.
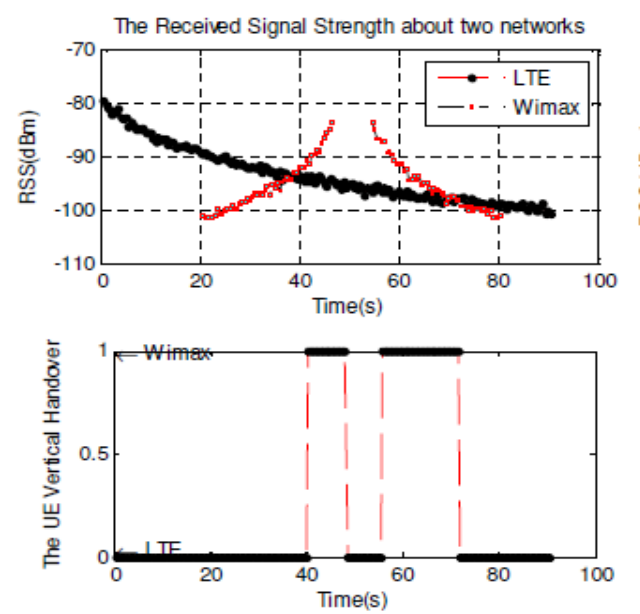

Figure 4. VH using the RSS based method

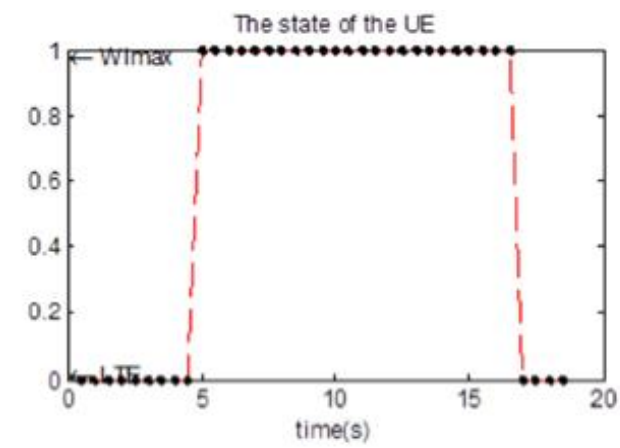

Figure 6. The UE handover using GAfVH based throughput

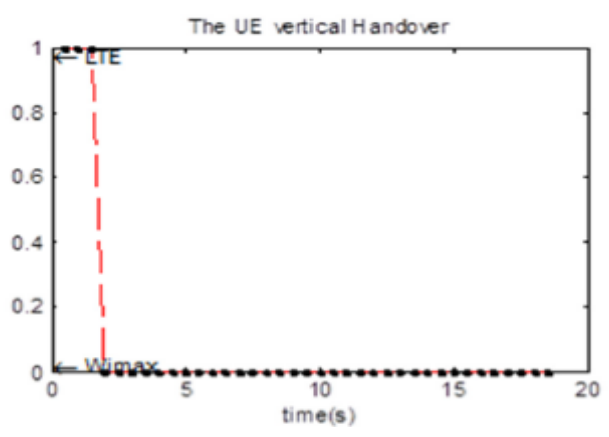

Figure 8 . The UE handoff using the proposed vertical handover (ber case)
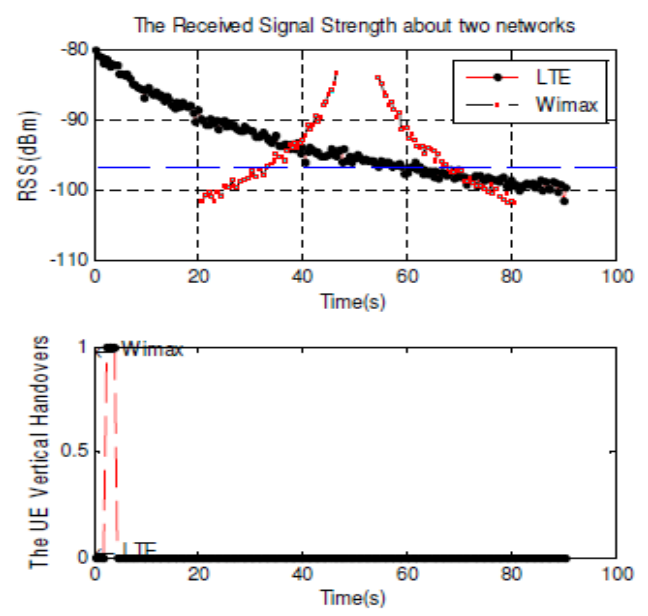

Figure 5. VH using the GAfVH algorithm

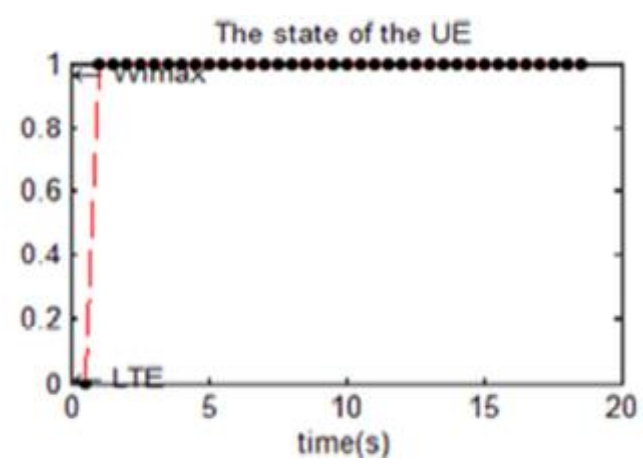

Figure 7. The UE handover using GAfVH based throughput calculation (case of a high number of users)

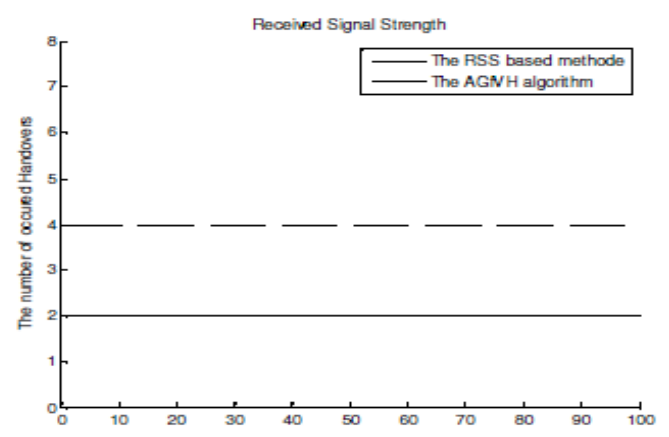

Figure 9. The number of the occurred handovers 


\section{CONCLUSION}

Our proposed algorithm is giving better and more accurate results readings, due to the simplified and optimized selection criteria process. Further more in the case of the applications usage the algorithm shows significant and positive results, which will also enhance the performance and reliability stats of this application.

\section{REFERENCES}

[1] K. Ahuja, et al., "Network Selection Based on Weight Estimation of QoS Parameters in Heterogeneous Wireless Multimedia Networks," International Journal of Wireless Personal Communications, vol/issue: 77(4), pp. 3027-3040, 2014.

[2] Q. N. Vuong, et al., "Multi-Criteria Optimization of Access Selection to Improve the Quality of Experience in Heterogeneous Wireless Access Networks," IEEE Transactionsof Vehicular Technology, vol/issue: 62(4), pp. 1785-1800, 2012.

[3] V. A. Narayanan, et al., "An Intelligent Vertical Handover Decision Algorithm for Wireless Heterogeneous Networks," American Journal of Applied Sciences, vol/issue: 11(5), pp. 732-739, 2014.

[4] R. Verma and N. P. Singh, "GRA Based Network Selection in Heterogeneous Wireless Networks," International Journal of Wireless Personal Communications, vol/issue: 75(2), pp. 1437-1452, 2013.

[5] I. Chamodrakas and D. Martakos, "A utility-based fuzzy TOPSIS method for energy efficient network selection in heterogeneous wireless network," Applied Soft Computing, vol. 12, pp. 1929-1938.

[6] H. Pervaiz and J. Bigham, "Game theoretical formulation of network selection in competing wireless networks: An analytical hierarchy process model," The Third international conference on next generation mobile applications, services and technologies.

[7] B. Liu, et al., "AHP and Game Theory based Approach for Network Selection in Heterogeneous Wireless Networks," IEEE Consumer Communications and Networking Conference (CCNC), Las Vegas, Nevada, USA, pp. 501-506, 2014.

[8] M. Lahby and A. Adib, "Network selection mechanism by using MAHP/GRA for heterogeneous networks," 6th Joint IFIP Wireless and Mobile Networking Conference (WMNC), Dubai, UAE, pp. 1-6, 2013.

[9] M. Lahby, et al., "A Novel Ranking Algorithm Based Network Selection For Heterogeneous Wireless Access," Journal of networks, vol/issue: 8(2), pp. 263-272, 2013.

[10] G. Wei, "Grey relational analysis model for dynamic hybrid multiple attribute decision making," Elsevier, Journal of Knowledge-Based Systems, vol. 24, pp. 672-679, 2011.

[11] A. Gharsallah, et al., "Network Selection in Heterogeneous Wireless System Environments," Journal of Networks, vol/issue: 10(12), 2015.

[12] C. R Reeves and J E Rowe, "Genetic Algorithms: Principles and Perspectives A Guide to GA Theory," AA Dordrecht, Kluwer Academic Publishers, 2003.

[13] P. N. Tran and N. Boukhatem, "Comparison of MADM decision algorithms for interface selection in heterogeneous wireless networks," $16^{\text {th }}$ International Conference on Software, Telecommunications and Computer Networks (SoftCOM), pp. 119-124, 2008. 